\title{
Rule-Based Conflict Management for Unmanned Traffic Management Scenarios
}

\author{
Abdulrahman Alharbi \\ School of Aerospace, Transport \\ and Manufacturing \\ Cranfield University \\ Cranfield, UK \\ abdulrahman.a.alharbi@cranfield \\ .ac.uk
}

\author{
Arturo Poujade \\ Amazon \\ Amazon -Spain \\ Spain \\ arturopg95@gmail.com
}

\author{
Dimitrios Panagiotakopoulos \\ School of Aerospace, Transport \\ and Manufacturing \\ Cranfield University \\ Cranfield,UK \\ d.panagiotakopoulos@cranfield.a \\ c.uk;
}

\author{
Konstantinos Malandrakis \\ Connected Places Catapult \\ Milton Keynes, UK \\ konstantinos.malandrakis@cp.cat \\ apult.org.uk
}

Antonios Tsourdos

School of Aerospace, Transport

and Manufacturing

Cranfield University

a.tsourdos@cranfield.ac.uk

\author{
Ivan Petrunin \\ School of Aerospace, Transport \\ and Manufacturing \\ Cranfield University \\ Cranfield,UK \\ i.petrunin@cranfield.ac.uk
}

\begin{abstract}
The growing use of Unmanned Aerial Vehicles (UAVs) operations will require effective conflict management to keep the shared airspace safe and avoid conflicts among airspace users. Conflicts pose high risk and hazard to human lives and assets as they ma may result in financial and human loss. The proposed rule-based conflict management model consists of three main stages. The first stage includes strategic deconfliction during the flight plan generation. The second stage, pre-tactical deconfliction, applies a ground delay to the agent to resolve the conflict. The third stage corresponds to the tactical deconfliction, where the drone hovers or loiter in the last waypoint before the conflict area until the conflict time window passes. The proposed method differs from most existing conflict management approaches in that it applies deconfliction methods sequentially using a rule-based strategy. Furthermore, a high number of published studies do not consider realistic airspace constraints and potential airspace modernization concepts such as dynamic flight restrictions Assessment and validation are performed in three simulation scenarios that consider different patterns of the airspace availability in the areas where flights may be restricted, such as airfields, recreational areas, and prisons. The Particle Swarm Optimization (PSO) algorithm was used for drone path planning. For the simulated scenarios all of the conflicts were resolved after implementation of the proposed method. The implemented method is simple, flexible and suitable for the management of more complex and dense airspaces.
\end{abstract}

Keywords-UAV, No-fly zones, Conflict Detection and Resolution, Simulations, Time-based

\section{I.INTRODUCTION}

UAVs are revolutionizing many of our daily tasks including transportation, logistics, and surveying [1]-[2]. This is because UAVs open the door for new countless opportunities, such as reducing risks and costs [1], in many real-world applications including the aforementioned, and therefore there will be an increasing trend of UAV deployments [2]. Thus, the low- altitude air traffic is expected to rise considerably in the years to come. This fact has been further emphasized by [3], stating that the UAV flight origin and destination might be virtually everywhere. To quote Dr. Parimal Kopardekar in the open talk at DASC 2016: "every home will have a drone and every home will serve as an aerodrome" [4]. One of the prerequisites for the fruitful and safe real-world deployment of UAV fleets is the development of a safe and efficient Unmanned Aircraft System (UAS) Traffic Management (UTM) system [5]. There is a dire need to perform such services with safety and thus the path of each UAV must be free from static obstacles such as elevated terrains and no-fly zones. There is also a need to take care of dynamic obstacles such as other UAVs. Conflicts can result in a wide range of negative impacts both in the air, such as delaying or not delivering a service, obstructing airspace from other users and ultimately collisions, and on the ground fatalities. A conflict is defined as the predicted loss of both horizontal and vertical separation between two or more aerial vehicles [6]. It is necessary to develop an efficient conflict management system that must be able to detect conflicting traffic in sufficient time and perform avoidance maneuvers to resolve conflicts [7]

In UTM, and similar in Air Traffic Management (ATM) Conflict Detection and Resolution (CDR) methods refer to different layers of "redundancy" (Fig.1) as suggested by the International Civil Aviation Organization's (ICAO) Global Air Traffic Management Operational Concept [8]. The first layer is Pre-Flight CDR, where off-line conflicts are spotted and solved based on flight plans submitted to the UTM, before the actual flights. The second one is In-flight CDR, that also considers changing weather conditions, or some real-time emergencies. Thus conflict-free paths created by pre-flight CDR might not be safe anymore. Therefore, in-flight CDR methods help to moderate UAVs movement in real-time by providing conflictfree paths. 


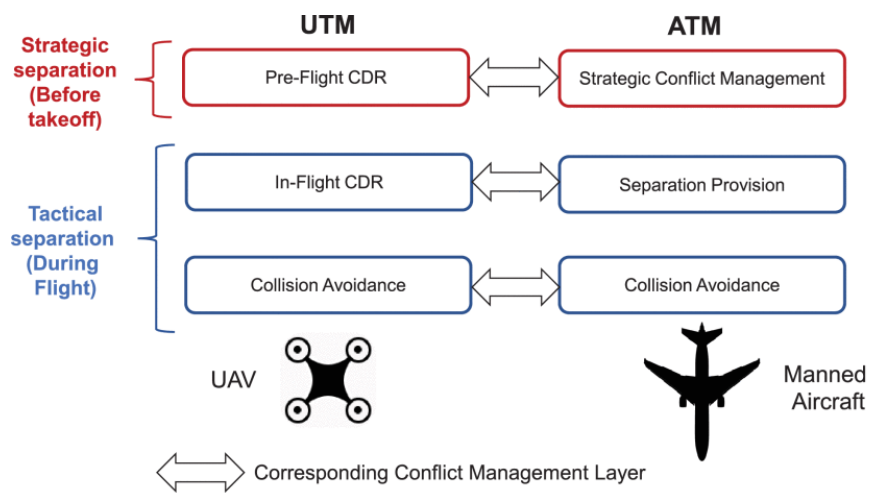

Fig. 1.Conflict management layers in ATM and UTM [2].

Recent works have proposed several separation methods, such as 4D (x,y,z \& time) trajectories based pre-flight airspace reservation method [2], modelling of the pre-flight conflict detection and resolution as a Multi-Agent Path Finding problem [9], implicit coordination of drone maneuvers and use of combined navigation and swirling functions [3]. However, these methods are computationally complex and generally appropriate for pre-flight deconfliction. These methods have limited scalability and also require additional (strategic and tactical) deconfliction to provide required safety level.

The work described in this paper adds novelty to this area by evaluating the effects of dynamic environments on the number of conflicts in densely populated air traffic scenarios. It also proposes and studies the efficiency of a three-layered deconfliction technique that encompasses rerouting, ground delays and hovering methods.

The rest of the paper is organized as follows: Section II presents related work and provides some background on the techniques used in this paper. Section III presents the simulation setting and considerations. Section IV presents the three simulations scenarios. Section $\mathrm{V}$ shows the deconfliction models proposed and applied to the conflicts in this dense airspace environment. Section VI presents the application of deconfliction. Section VII presents the deconfliction results discussion and analysis. Section VII concludes this paper and provides guidelines for future work.

\section{RELATED WORK}

\section{A. Path planning and optimization}

The occupancy of airspace by UAVs is determined by their flight paths that are established through a two-step process: first, task allocation decides on the sequence of destinations for the UAV (and associated actions), and second the path planning designs of an optimal trajectory under the terrain and airspace constraints.

The primary goal in path planning is to create the shortest possible path with collision avoidance. The field of robotics has since long studied this problem. The work in [12] uses the concepts of artificial time-varying potential fields to provide collision-free trajectories. Others have studied the elastic band properties to provide a short and smooth obstacle-free path [10]. However, those methods are limited to low-dimensional problems and cannot deal with complex constraints. Samplingbased motion planning algorithms such as Rapidly exploring Random Trees (RRT) that consider complex constraints have also been proposed in [11]. Although these approaches produce trajectories that fulfil constraints, the trajectory is not optimum and the computation time exponentially increases as the order of the search space increases.

Optimization techniques are also being utilized to address motion planning problems. The work in [12] presents an approach using a stochastic trajectory optimization framework. The basis of this work lies in spawning noisy trajectories to discover the space around an initial trajectory that are combined to update a trajectory with low cost. The algorithm is subjected to smoothness and obstacle constraints to produce an optimized collision-free trajectory. Although stochastic optimization methods have a fast convergence rate, a high success rate and can include various constraints, yet these methods optimize individual steps and may converge to a local minimum rather than the global minimum.

In this study, the trajectory optimization using PSO is investigated. PSO is a population-based stochastic optimization method inspired by the behavior of wildlife in groups such as flocks of birds or schools of fish [13]. The main advantages of PSO are that it is simple to understand, easy to implement and converges rapidly compared with other traditional global optimization methods such as genetic algorithms and simulated annealing [14].

\section{B. Conflict Detection \& Resolution Methodlogies}

Several studies have been done that contribute to the evolution of a safe and secure UTM system for high-density low-altitude airspace. The work in [15] proposes to use selective velocity obstacle method in tactically de-conflicting the UAVs in real-time. The study in [16], proposes an adapted optimal reciprocal collision avoidance approach that takes care of practical considerations such as navigation inaccuracies, communication overhead, and flight phases in order to state minimum separation distances for a conflict-free zone. It is thus evident that the above approaches consider the integration of inflight CDR methods only.

Apart from these, other researchers have focused on sense and avoid methodologies. For example, in Agent Fly project [17], the sense and avoid function uses intelligent algorithms and a communication channel provided by onboard wireless data modems and sensory data providing information about objects in its surrounding such as onboard radar system or transponders. The work in [18] suggests a reciprocal collision avoidance algorithm based on the velocity obstacle approach that guarantees collision-free maneuvers even when the agents are only capable to sense their environment within a limited field of view. A classification of CDR approaches is presented in [16] in order to define their role in overall safety management of UAV operations in integrated airspace. This classification is based on the type of surveillance, co-ordination, maneuver and autonomy of UAV missions. Keeping in view the above taxonomy, this work further elaborates a multilayered architecture that encompasses procedural, manual, cooperative, non-cooperative, escape and emergency methods applied in a 
layered fashion. The work in [19] address the requirements of airspace to be made flexible and dynamic in view of airspace demands, equipage and weather conditions. They propose three core areas to look into specifically: restructuring airspace, adaptable airspace, and generic airspace environments. They also present mid-term and long-term airspace configuration concepts that include high altitude airspace and low altitude airspace regions for high density. This study may help in devising any future CDR methodologies for UAV dynamic airspace environments. The work in [3] present different conflict resolution schemes, for low altitude uncontrolled airspace and estimated the capacity under various airspace management scenarios. They evaluated various conflict resolution methods, including ground-delay, hovering, layered assignment and ondemand, descend for single-layer airspace where all air-vehicles occupy the same altitude and also multilayers which consider the horizontal layers at altitude level below or above. The proposed methods were validated and compared using simulations. The study in [17] address the use of priority-based planning to avoid collisions where each robot is assigned a unique priority. The trajectories for individual robots are then planned sequentially from the highest priority robot to the lowest priority one to avoid collision with static obstacles and other robots.

Several ATM schemes have been studied that may lay down a path for de-conflicting UAVs. The work in [20] addresses the use of prior flight level allocations and ground holding strategies for ATM scenarios. They suggested to use a prior flight level allocation program to reduce the complexity of air traffic flow before applying the ground holding algorithm to solve all conflicts and thus improving the quality of solutions. A study in [21], also uses the shifting in departure times or ground delays for solving ATM conflicts. However, their methodology has been able to experience results into an average departure time shift of 21.6 to 23.2 minutes with a maximum of 60 minutes delay that will add additional cost to UAV traffic agencies. This work addressed the problem of rerouting by constructing an alternate trajectory with the help of M-virtual waypoints along the nominal trajectory in the horizontal plane that were later reconnected using straight line segments. The path optimization was done using a simulated annealing algorithm. The European Network Manager Operations Centre suggest different strategies to deconflict whenever an en-route sector is overloaded, such as better ground holding, flight level allocation and assignment of alternate trajectories [22]. This work has also shown ground delays of the order of 30 to 60 minutes for resolving conflicts but suggests a fairness factor assigned to conflicting UAVs. The objective is to minimize the delays, financial loss and environmental impact of the congestion. A ground delay approach is presented in [23] where all adjustments are imposed in the departure times to avoid the conflicts. Furthermore, the authors present an approach to account for uncertainties, which unfortunately results in high delays. The work in [24] explores the implications of decentralized trajectory deconfliction in UTM on fairness. The authors used simulation as a tool to explore how a First-come, first-served approach to strategic deconfliction in UTM performs in terms of fairness. Fairness is quantified by comparing average ground delay across operators and by calculating a normalized fairness metric that accounts for operator cost of delay. The idea of pre-flight rerouting and filling service presents in [25] where filling service is making sure that new routes do not conflict with other trajectories. The work in [5] employs the hovering capabilities of drones. UAV departures are not delayed, but instead, the drones wait in the air by hovering on a lower altitude until the current conflict is resolved. In [3] the authors address the deconfliction strategies for UAV drones that have hovering capabilities. They make the conflicted UAV with lower priority to hover at low altitudes before the conflict passes away. Although independent de-confliction strategies do exist, yet there is a strong need to provide a seamless operational flow by the combination of different schemes.

\section{Description of the Particle Swarm Optimization}

In 1995, the technique of PSO was first pioneered by James Kennedy and Russell Eberhart [19]. Since then, it has been widely used for addressing intricate optimization issues and had been acknowledged for its efficiency recently. The major applications of this technique are illustrated in character recognition, power appropriation of cooperative communication networks, machine learning, and evolving artificial neural networks among others [26]. In order to identify the best probable solutions that correspond to the optimization challenges, this technique involves persistent movement of the particles within the search process. There are three derivatives of the algorithm function:

Optimization: The movement of the particle is contingent upon the swarm intelligence, best solution candidate and velocity when the optimal swarm position is achieved

Collaboration: The information about the best solution is exchanged between the particle and the swarm, which is followed by the consideration of optimal solution by the swarm to the defined objective.

Adjustment: The adjustment of each particle is dependent upon its movement in three vectors. The random scalar time for each particle is noted when it moves between the local position and the optimal position of swarm while utilizing the personal inertia. The particle illustrates this movement repeatedly while coinciding with the number of simulations running in parallel. The particle reaches closer to the global best solution after every movement.

In addition, PSO can be leveraged to count and determine the optimal values of trajectories of from A to B. In so doing, PSO identifies the best possible trajectory which is costeffective and efficient against any challenge. Since the PSO algorithm was consumed for anticipating and planning drone path, it was particularly helpful to identify the solution that requires the minimum movement between starting and endpoints while avoiding any obstacles.

Few of the preceding studies have suggested hierarchical phased deconfliction strategy for UAV regime using rerouting, ground delay and hovering. This work attempts to present a more realistic picture by associating priorities to various UAV missions and applying these strategies phase-wise to achieve fine deconfliction efficiencies. It is observed that as the traffic grows the pre-flight level allocation has been unable to solve the growing high-density conflicts [2]. Furthermore, this study 
presents the need of multi-stage deconfliction by applying the independent strategies one after the other to resolve conflicts.

\section{Simulation Setting \& CONSIDERATIONS}

The proposed method is assessed and validated through simulation that represents the airspace over Bedfordshire and Buckinghamshire in the UK and uses nine areas where flights may be restricted, such as airfields, recreational areas, and a prison, as illustrated in Fig. 2. These are:

1. Four airfields, including Luton, Cranfield, Halton and Old Warren (orange);

2. Four recreational areas, including Dunstable, Sandy, Cardington and Graveley (yellow);

3. Milton Keynes Prison (blue).

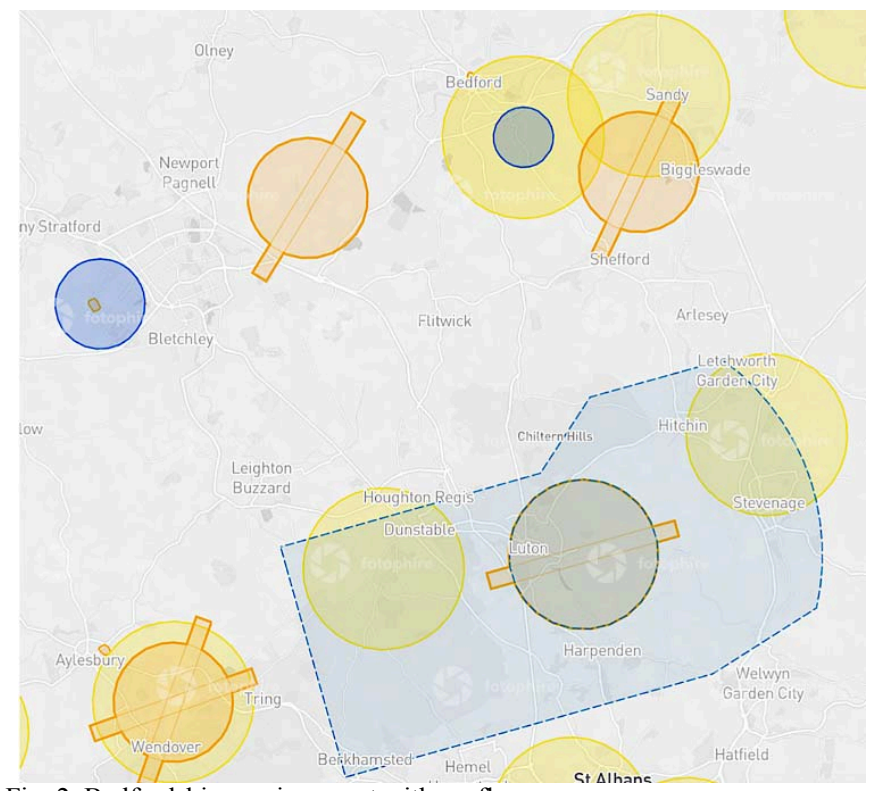

Fig. 2. Bedfordshire environment with no-fly zones.

The following simulation settings were considered that set the limitations and requirements, and making things more realistic and closer to a real-life scenario:

- Random environment's availability: In order to consider the dynamics in environment, the availability of recreational areas and dynamic airfields are set randomly during the second and third hour of simulation scenarios. The areas, number of minutes which disappears and not constant are selected randomly.

- Random number of flights per hour: A random number of flights is set to fly per hour in order to force the system to have some conflicts. This number has been varied between 30 to 40 .

- Random departure times: To make the system more realistic, the exact time each agent departs is set randomly.
- Period of simulation runs: The duration of each scenario has been set to one hour.

- Random start and finish locations: The simulation has been set to make the locations of the start and finish points to be selected randomly. Since the simulations have been done considering the location map of the whole Bedfordshire area in U.K., each flight departs and finishes at a random point.

- Random priority levels: Each flight has been set with a random priority level of service from level 1 (highest priority) to level 5 (lowest priority). This random prioritization will be used in our deconfliction strategies.

- Constant UAV velocity: The velocity of each UAV agent is considered as constant and the same for all UAVs.

\section{SimUlation ScEnARIOS}

Three simulation scenarios were run in MATLAB for the Bedfordshire environment explained earlier. The number of UAVs flying per hour in these simulations ranges between 30 40. Trajectory data is a set of waypoints defined as:

$$
W_{i}=\left(x_{i}, y_{i}, t\right)
$$

where, $x_{i}$ is the coordinate in the $\mathrm{x}$-axis, $y_{i}$ is the coordinate in the y-axis, and $t$ is the exact hour the agent is passing by that waypoint. A conflict is defined when two or more agents are at the same coordinates at the same time.

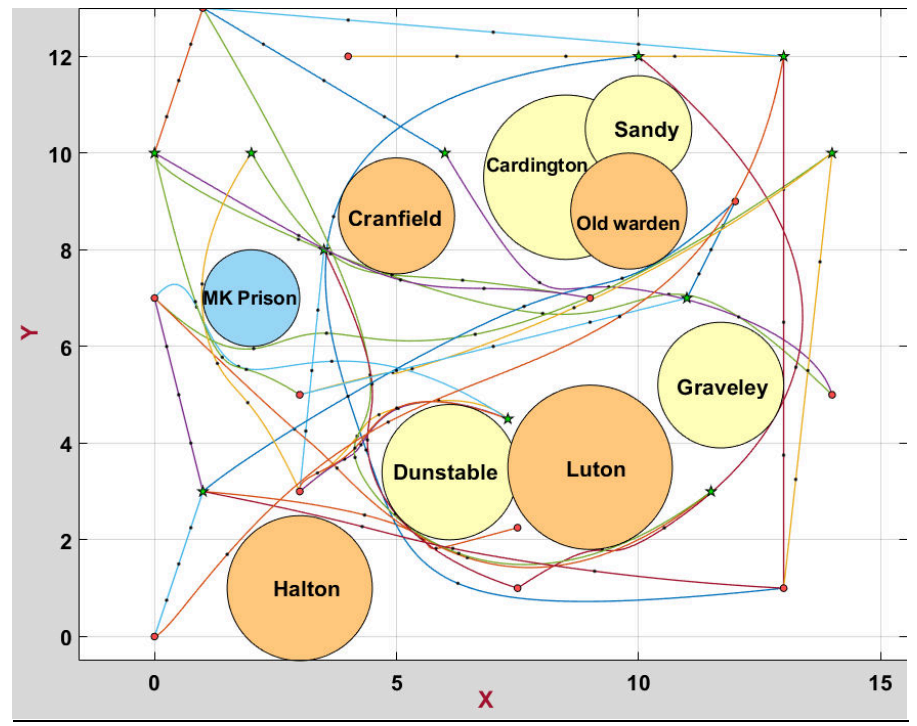

Fig. 3. Simulation example for 32 UAVs.

\section{A. First Hour of Simulations - Static No-Fly Zones}

The first hour runs between 8 am and 9 am. During this period UAVs were not allowed to fly over the nine obstacles defined that remained static and didn't open up their airspaces. A total of 32 flights were scheduled during this hour of operations and all of these flights departed and landed at random locations. The PSO based algorithm found a feasible path solution for all the demanded trajectories. During this, period, 14 conflicts were found. The conflict heat map is presented in Fig. 4. 


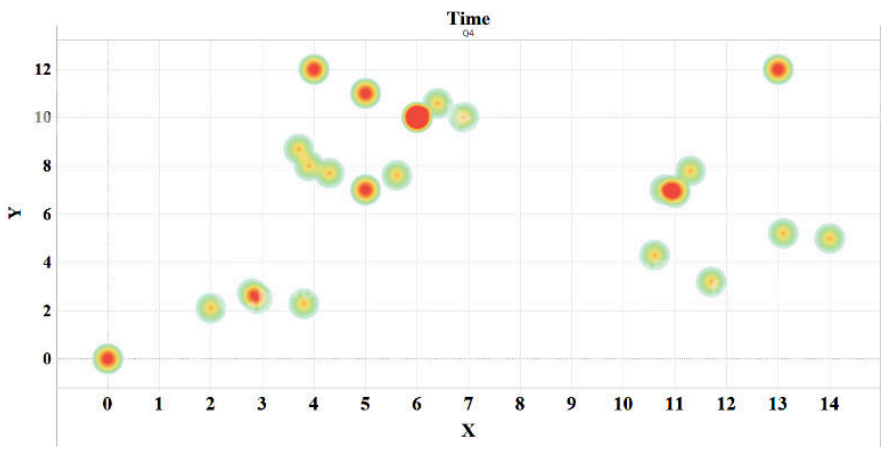

Fig. 4. First hour simulation - conflict heat map.

\section{B. Second Hour of Simulations - Dynamic Recreational Areas}

The second hour of simulation runs between 9 am and 10 am. The associated heat map is shown in Fig. 5. The difference with the previous simulation is that the no-fly zones corresponding with the recreational areas are dynamic. This dynamism is brought about by making the availability and unavailability of these areas random during the whole hour. Therefore, some agents could fly over these areas at some point between this hour. Also, the recreational areas of Cardington and Graveley opened their airspaces. A total of 39 agents were simulated during this hour. During this, period, 26 conflicts were found. The conflict heat map is presented in Fig. 5

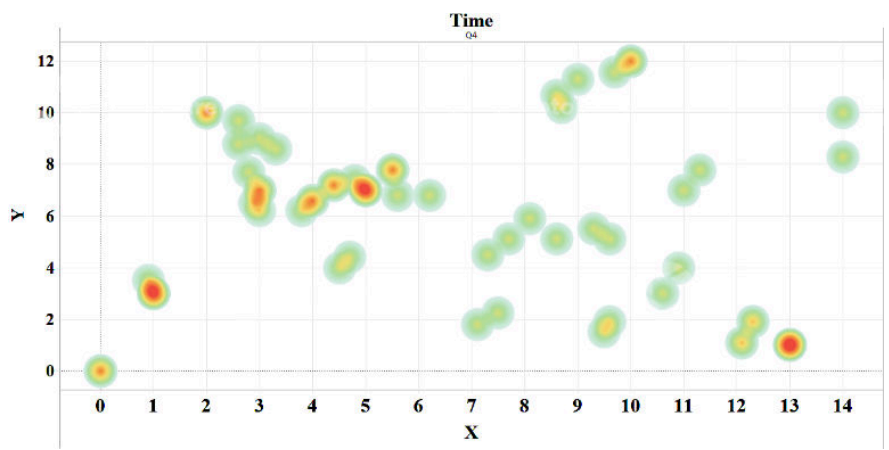

Fig. 5. Second hour simulation - conflict heat map.

\section{Third Hour of Simulations - Dynamic Recreational Areas}

The third hour of simulation runs between 10 am and $11 \mathrm{am}$. The heat map is shown in Fig. 6. The difference with the previous simulation is that the no-fly zones corresponding with airfields are dynamic. This dynamism is brought about by making the availability and unavailability of these areas random during the whole hour. Therefore, some agents could fly over these areas at some point between this hour. Also, the Luton and Old Warren's airspace are opened for traffic. A total of 35 flights were simulated during this hour. During this, period, 18 conflicts were found.

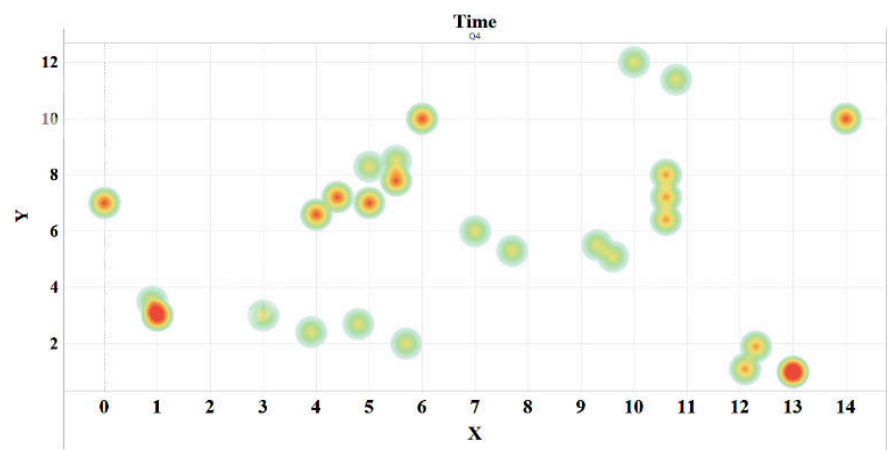

Fig. 6. Third hour simulation - conflict heat map.

Another key figure of merit has been created in this work that corresponds to the conflicts per hour of simulation and the number of agents in sky at that time. Using three hours of simulation data, the above statistics have been presented below in Fig. 7. In the vertical axis, we observe the number of conflicts, whereas in the horizontal axis we have a time scale. The size of each marker and the numbers enclosing the markers denote the count of agents (1-20) flying at that specific moment.

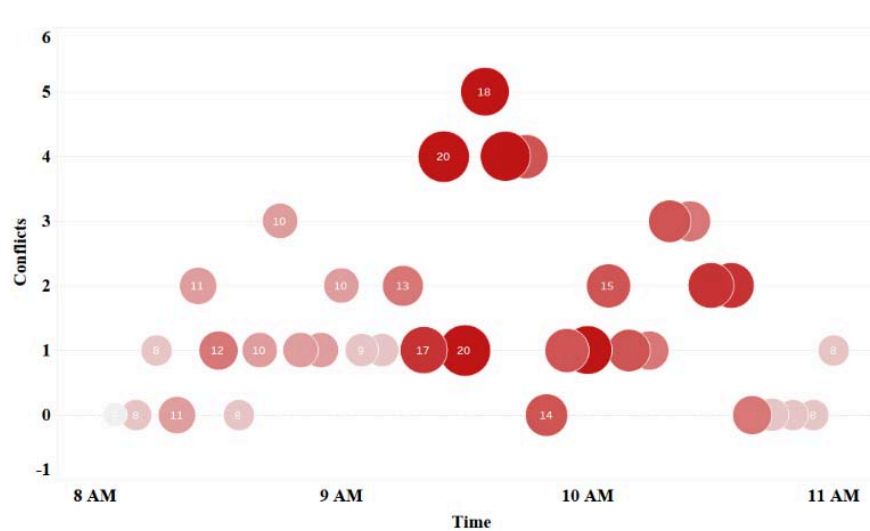

Fig. 7. No of conflicts and agents in the sky from $8 \mathrm{AM}$ to $11 \mathrm{AM}$.

A trend analysis has also been conducted based on the number of flights and conflicts for three hours of the simulation as shown in Fig. 8. It can be inferred from both (Fig. 7 \& Fig. 8) that the greater the number of agents in the sky greater the chances are of having a conflict, and also there is a possibility of more than one conflict at a particular moment in time as we have observed a maximum of 5 conflicts at the same time.

It is also observed that as the number of flights are added in the airspace environment for all three hours of simulations, the number of conflicts grow in a polynomial pattern.

An important correlation exists with the change in the environment in 2nd and 3rd hour of simulations where airspace environment was opened for some recreational areas and airfields. It has shown less rate of increase in conflicts in comparison with 1 st hour of simulation where the airspace remained closed for all dynamic recreational areas and airfields. This seems a more realistic picture in the real world. The rate of conflict tends to decrease with the availability of more area to 
fly whenever some dynamic areas are opened up for flying over them.

From Fig. 8, It can be seen that between 9:20 to 10:40 hours of simulation, there has been steady increase in the number of flights resulting in some regions of conflict peaks. This increase is due to presence of more UAVs in the air from pervious hours and new incoming flights and also their interacting trajectories.

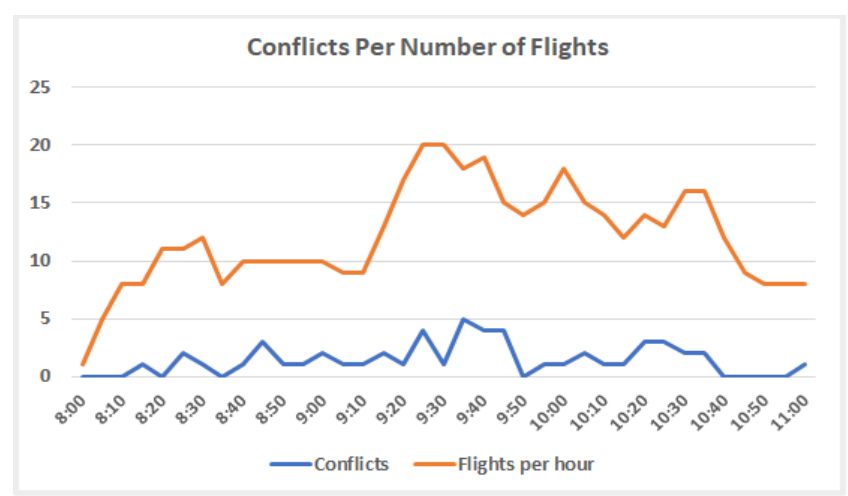

Fig. 8. Conflicts per number of flights.

From the first hour of simulation trajectory and heat maps, it can be seen that the corridor between two zones especially $(3,7)$ and $(11,7)$ is providing the airspace for most flights to pass through that increases the chances of conflicts and resulting in red hot conflicted zones where more than two UAVs conflict at the same time.

The second hour of simulations allowed the airspace above the recreational areas of Cardington and Graveley open for flying over. This resulted in more space to fly over and thus caused an evenly spread out of the conflicts in this comparatively wider corridor in contrast to 1 st hour simulations. This phenomenon is visually apparent as there are less red-hot areas in the heatmaps. The same phenomenon has been observed in the third hour simulation data also where Luton and Old Warden also opened up their airspaces. However, some new orange heatmaps or less conflicted zones does show up due to the addition of more incoming and already prevailing flights in the airspace during these later simulation hours.

\section{DECONFLICTION METHODLOGY}

The next section discusses the deconfliction models proposed and applied to the conflicts in this dense airspace environment, and the goal is to strategically deconflict any number of trajectories within the same environment. An important consideration in dealing with conflicts with multiple agents involved at one time is that deconfliction is applied to one with least priority level. The proposed deconfliction model comprises of three different stages. We propose to apply each stage before departure and during UAV flying.

\section{A. Deconfliction Model First Stage}

The first stage (Fig. 9) is applied ideally days before departure. This stage constitutes of two different methods, preflight rerouting and filling service. Pre-flight rerouting is changing the route when we have two trajectories on the same point at the same time, avoiding this conflict zone. Filling service validates if this new route doesn't conflict with other trajectories.

The proposed model combines these methods in a seamless operation flow to enhance deconfliction in-order to cater more complex and dense traffic operations. The flow chart for this stage is presented below and will be connected to the rest of the chain in subsequent sections.

This approach either modifies the Extended Flight Plan (EFPL) for all the conflicts in a multilayer (based on two designs of the airspace: the single-layer airspace, where all UAVs occupy the same altitude, and multilayers that UAVs have different altitude capabilities [3]), or would result in a cancellation of existing EFPL, if conflicts still exist, and demand submission of a new EFPL.

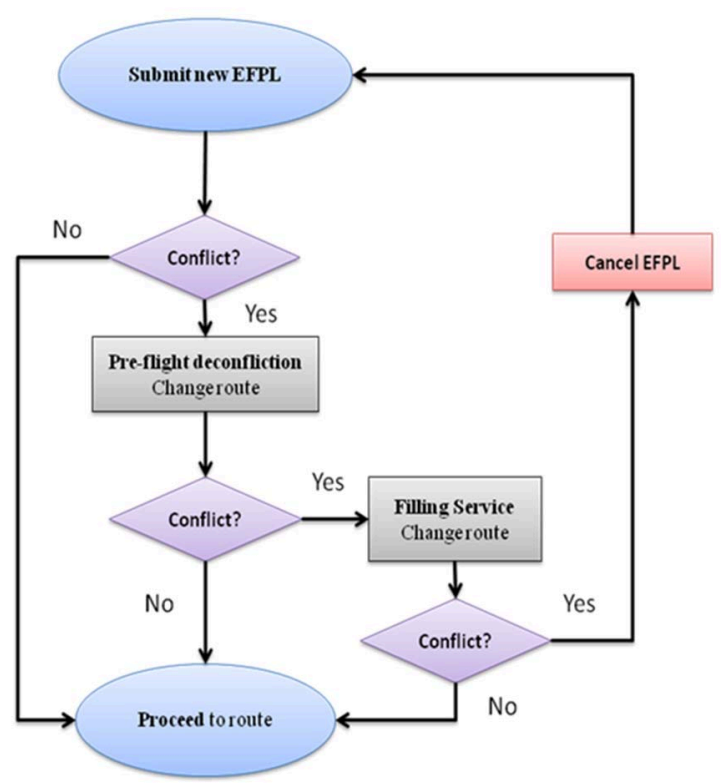

Fig. 9. First stage of strategic deconfliction.

\section{B. Deconfliction Model Second Stage}

The second stage of deconfliction as shown in Fig. 10 is achieved by applying ground delays to UAVs with less priority before departure. The maximum delay could span between two to three minutes on the ground, similarly, that it is currently being done in ATM. The proposed process flow is divided into two branches, one for a single layer of airspace and the other branch applied to multiple layers of airspace.

The ground delays are introduced in case of conflicts in single or multilayers of the order to max delay time till all the conflicts are resolved or otherwise results in cancellation of EFPL. In case no conflict exists, the agents are allowed to proceed as per their routes. 


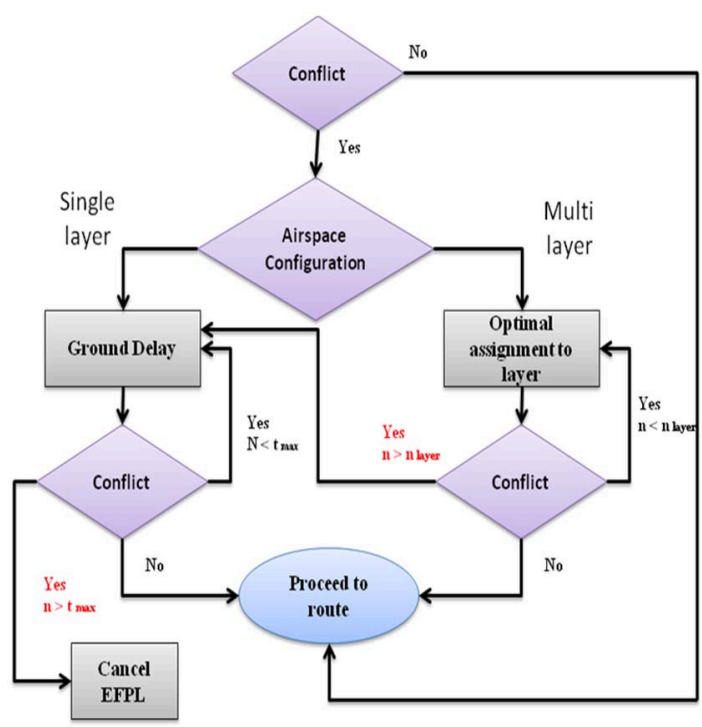

Fig. 10. Second stage of pre-tactical deconfliction model.

\section{Deconfliction Model Third Stage}

Hovering is the third stage of deconfliction applied just about the departure time as depicted in Fig. 11. The process of hovering starts at a waypoint preceding the conflicted zone. This introduces a pause in the process until the conflict is resolved. This stage highly depends on one factor; battery endurance of the UAV. Battery time is the biggest constraint when applied to any UAV and needs careful consideration before adoption. Secondly hovering at a previous waypoint is a solution that only rotary-wing agents could adopt, making those UAVs more manoeuvrable and easier to deconflict. However, for fixed-wing $\mathrm{UAVs}$, it is recommended to allocate them higher priority levels.

The hovering is applied in case of conflicts in single or multilayers. In an event of conflict, the UAVs in the air are subject to hovering at the last waypoint till the expiration of safe battery time (tbat) or land otherwise if this time expires. If the conflict is resolved the other agents in this conflict are allowed to proceed to their designated routes.

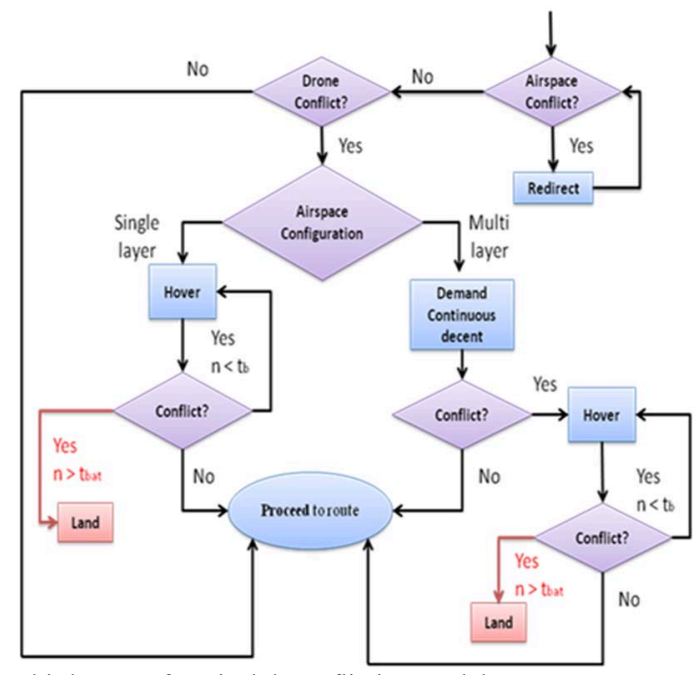

Fig. 11. Third stage of tactical deconfliction model.

\section{VI.APPLICATION OF DECONFLICTION}

In order to demonstrate the proof of concept, the methodologies described in the previous section were applied to 58 conflicts that appear during the simulations. The next section will present the results of reroute, ground delay and hover strategies with the help of examples.

\section{A. Rerouting}

Rerouting is the primary method of deconfliction proposed here that is based on modifying the route of the conflicted UAV days before the flying mission. It is also suggested to apply this strategy to UAVs with less privileged tasks or priorities. In the example below, a conflict between two independent airspace users is resolved through rerouting. These trajectories were uploaded as EFPLs by two different airspace users. The first user wants to fly from point $(0.0)$ to point $(10,12)$ (blue line), and the second user wants to fly from point $(0,7)$ to point $(5,1)$ (orange line) (Fig. 12).

Orange user departs 30 minutes after the blue user. It can be seen that both trajectories cross in point $\mathrm{Q}(\mathrm{x}, \mathrm{y})=(2.62,3.84)$ at the same time (43.1). Nowadays, the UAV traffic numbers are increasing, and the complex UTM operations are becoming a reality. Fairness and equity are essential in the UTM framework of the ICAO, which states that "access to the airspace should remain equitable" [27]. To deconflict firstly both users' priority level needs to be checked. Blue user has less priority level, and therefore it is required to be rerouted. To reroute one's trajectory, a virtual obstacle has created to the trajectory that needs deconfliction at the point of conflict. The conflict area, which is represented by a red circle in Fig. 13, shows the avoidance mechanism of algorithm with a new deconflicted green route.

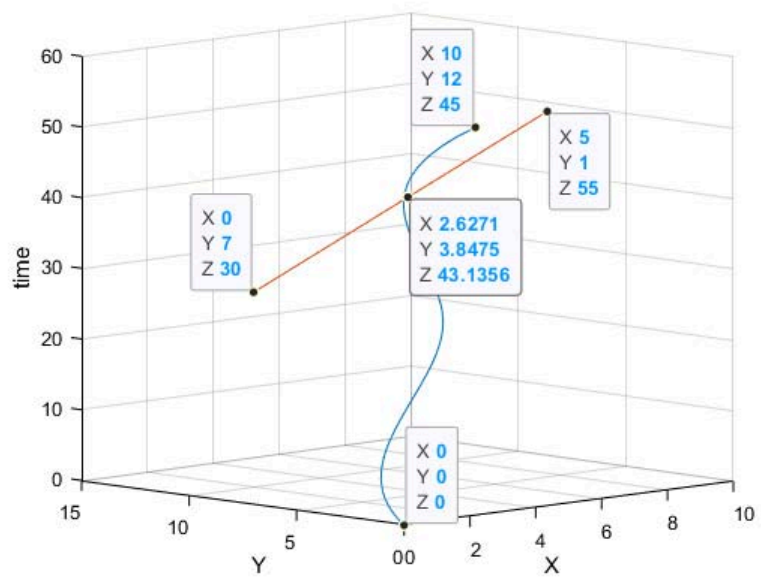

Fig. 12. Rerouting Example: Confliction case. 


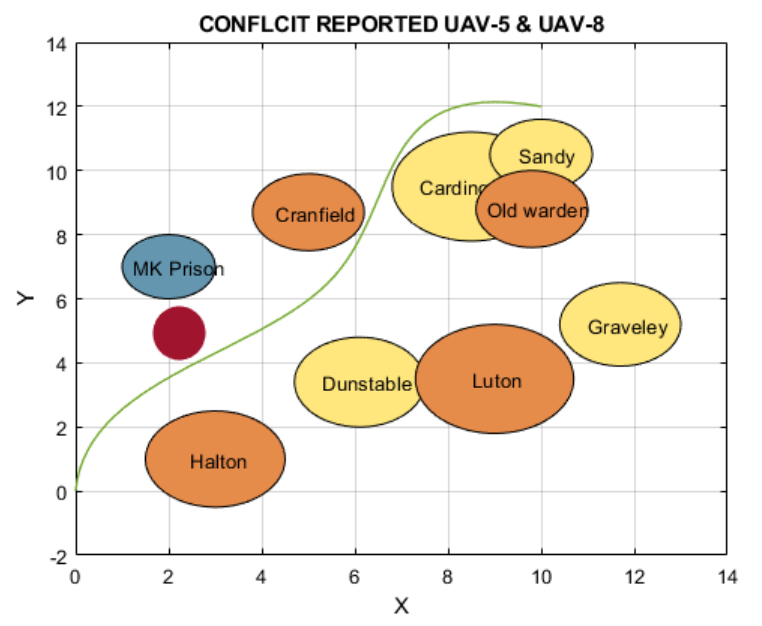

Fig. 13. Rerouting Example: Deconfliction case.

Moreover, an assessment is performed to establish if this new trajectory conflicts with another agent. This process of filling service works the same way as explained for the first rerouting service. The deconfliction method applied in this scenario resolved the problem as both UAVs can cross the point of conflict at different timings thus avoiding conflict.

\section{B. Ground Delay}

The second layer of deconfliction method is mainly applied a few hours before departure. Assuming that there are two UAV flight plans: the first trajectory is planned three days before departure, and the second trajectory is an emergency operation, that is shared twenty minutes before departure. Both flights have a conflict at the same time in the same point. In this case, rerouting was not planned. Therefore, a ground delay of 3 minutes is applied to the least priority flight This resolves the conflict and assures that a new conflict does not appear due to this ground delay.

\section{Hovering}

The third layer of the deconfliction method proposed in this work is hovering. When none of the above deconfliction strategies are valid to a specific situation, hovering is the last option. This technique is limited to rotary-wing agents and is dependent upon critical factor considerations, such as battery life. The battery endurance depends on maximum take-off weight, battery capacity, planned trajectory/flight time, and so forth. However, in this study we do not consider it in the simulation due to simplicity of the simulated scenario. Hovering maintains a UAV static for 2 minutes, waiting until the conflicted trajectory passes.

Overall, with this proposed layered deconfliction methodology, the solution was able to deconflict every flight in a strategic phase for all 58 conflicts as shown in may observe in Fig. 14.

In the first hour (8:00-9:00) of simulation with no-fly zones, a total of 32 agents were simulated during this hour, 14 conflicts were resolved followed by 26 in the second hour (9:00-10:00) A total of 39 flights were scheduled during this hour of operations with dynamic availability of recreational areas. All 18 conflicts were addressed in the third hour (10:00-11:00) of the simulation were 35 flights simulated during this hour. Since the airspace is heavily loaded with a greater the number of flights between 9:20 to 10:20 hours, we have observed a greater the number of conflicts during this period. Rerouting mechanism has been the most successful method to deconflict the airspace in these scenarios followed by ground delay and hovering.

As already discussed in Section V (simulation scenarios), the airspace was narrow during first hour of simulation due to airspace restrictions thus providing less room for rerouting, however, 2nd and 3rd hour of simulations provided more airspace volume to apply rerouting mechanism as they opened some of their airspaces dynamically for flying.

It has also been observed that ground delay has been the second most successful method as hovering comes with additional constraints of battery capacity. Also, the presence of a static UAV in the air would add to the complexity of airspace by hindering other flight paths making deconfliction difficult as compared to ground delays.

More studies with different traffic volumes and environments would be highly practical to see how traffic volume affects the strategic deconfliction.

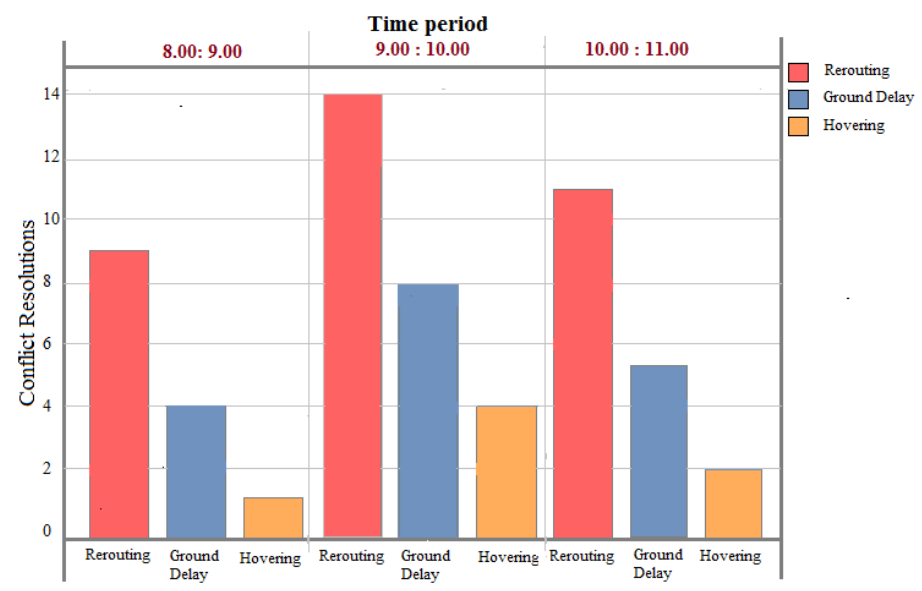

Fig. 14. Conflict resolution by process.

\section{DeCONFLICTION METHODOLOGY RESUlts DisCUSSION}

The proposed deconfliction methodology has shown highend gains by solving all the conflicts in a layered fashion applying independent strategies one after the other. It has been observed that in the first hour of simulations data, the rerouting has been able to resolve $64 \%$ of all conflicts reported. Ground delays were able to resolve only $29 \%$ of total conflicts. The hovering mechanism helped resolving the remaining $7 \%$ of conflicts only.

The second hour of simulations data in dynamic recreational areas account to $54 \%, 31 \%$ and $15 \%$ resolutions by rerouting, ground delays and hovering, respectively. This statistic also remained closely true for third hour simulation data that showed $61 \%, 28 \%$ and $11 \%$ resolutions for all the three strategies respectively. 
As per airspace standards determining the prioritization of operations [27] (i.e., UAV flight with emergency having priority over other flight ). To reroute the UAV with the lowest priority in conflict, a virtual obstacle has creates with $0.5 \mathrm{~km}$ radius at the point of conflict. It has been seen that the proposed rerouting methodology has been able to solve almost $59 \%$ of conflicts arise during various static and dynamic environmental scenarios.

This work has also achieved a reasonable grounding delay ranging from 3-5 minutes to resolve conflicts arising in case of any emergency trajectory mission brought about some minutes before departure. This counts to about $29 \%$ of all conflicts.

Hovering of the order to 2 minutes wait time has been applied at a tertiary stage in the proposed methodology in the same horizontal space at a waypoint just before the point of conflict thus resolving about $12 \%$ of remaining conflicts.

The drawback of this approach is the additional power that could even lead to an emergency landing while waiting in airfields. In order to resolve this problem it suggested by allocate high priorities to low-energy drones while preventing their hover-like manoeuvres.

Few previous studies have provided an efficiency of a layered multi-stage deconfliction methodology applied on UAV regime, yet we can analyze the statistics with the ones in [20], that solves a pre-flight level allocation or rerouting to resolve the conflicts for a one day of European airspace data with residual conflicts solved by ground holding phase. There pre-flight level allocations or rerouting solved $65 \%$ of the conflicts and the remaining $35 \%$ were taken care by ground-holding phase. The results provide similar metrics, where rerouting resolved about (59\%) of the conflicts along with ground holding (29\%) and hovering (12\%) addresses the rest $41 \%$ of the conflicts for three hours simulation data. Their work has also shown a maximum ground delay of 3-5 minutes for almost $90 \%$ of their flights as shown in this study.

The potential downside of this study deconfliction strategy would be loss of flight efficiency. The flight would get delayed either due to a longer alternate flight path or delays due to stay on the ground or in air due to hovering. The study presents in [21] suggests to use an extension in travel time as one of the performance metrics in evaluating the efficiencies of deconfliction strategies. This metric also directly affects the operating costs of any travel plan. Since the most prominent rerouting strategy is based on modifying the path of single low priority UAV, it is assumed that this will not lead longer extensions in travel times for the other UAVs in the air.

\section{CONCLUSION \& FUTURE RECOMMENDATIONS}

It has been observed through simulations that, as the number of agents in the sky increase at some particular time slot, more conflicts are reported as we have observed a maximum of 5 conflicts at same time when a maximum of 18 agents were in sky. Based on the trend analysis, it has been observed that this increase follows a polynomial trajectory. The effects of introducing static and dynamic environments have shown that the rate of conflicts tend to decrease when dynamic areas are opened up as a result of more available space to fly pass. The efficiency of proposed three-layered deconfliction model based on rerouting, ground delays and hovering has been evaluated by applying these approaches to conflicts addressed above. It has been observed that rerouting is the most fruitful strategic deconfliction method that accounts for $58.6 \%$ of the resolution followed by ground delay that resolves $29.3 \%$ of conflicts. The hovering only accounts for $12.1 \%$ of resolutions.

It has also been seen that ground delays of the order of 3-5 minutes were sufficient to resolve some conflicts. The hovering time of 2 minutes has resolved remaining conflicts though in fewer percentages.

Finally, it will be commented how this project could be enhanced in the future, There have been ,several random inputs applied to the system, with the exception of the velocity of each agent. The speed of UAVs may be changed randomly to study the effect of changing speeds on the conflicted zones as future work.

Although, the agent or UAV localization model created and used in this project is two-dimensional 2D (X,Y), one avenue of future research is to add one more dimension i.e. $Z$ (altitude) to understand how one more degree of freedom would affect the system's performance. The effect of various environmental scenarios can be further studied by conducting more simulations, increasing the number of UAVs and bringing more dynamics areas.

\section{REFERENCES}

[1] H. Hildmann and E. Kovacs, "Review: Using Unmanned Aerial Vehicles (UAVs) as Mobile Sensing Platforms (MSPs) for Disaster Response, Civil Security and Public Safety," Drones, vol. 3, no. 3, p. 59, 2019.

[2] F. Ho et al., "Pre-Flight Conflict Detection and Resolution for UAV Integration in Shared Airspace: Sendai 2030 Model Case," IEEE Access, vol. 7, pp. 170226-170237, 2019.

[3] L. Sedov and V. Polishchuk, "Centralized and Distributed UTM in Layered Airspace," pp. 1-8, 2018.

[4] L. Sedov, V. Polishchuk, and V. Bulusu, "Sampling-based capacity estimation for unmanned traffic management," AIAA/IEEE Digit. Avion. Syst. Conf. - Proc., vol. 2017-Septe, 2017.

[5] F. Ho, R. Geraldes, A. Gonc, M. Cavazza, and H. Prendinger, "Improved Conflict Detection and Resolution for Service UAVs in Shared Airspace," IEEE Trans. Veh. Technol., vol. PP, p. 1.

[6] B. M. and N. A., "A Conceptual Framework and a Review of Conflict Sensing, Detection, Awareness and Escape Maneuvering Methods for UAVs," Aeronaut. Astronaut., no. May 2014, 2011.

[7] E. Calvo-Fernández, L. Perez-Sanz, J. M. Cordero-García, and R. M. Arnaldo-Valdés, "Conflict-free trajectory planning based on a data-driven conflict-resolution model," J. Guid. Control. Dyn., vol. 40, no. 3, pp. 615$627,2017$.

[8] ICAO, "Doc 9854, Global Air Traffic Management Operational Concept," Int. Civ. Aviat. Organ., p. 82, 2005.

[9] A. Salta, R. Geraldes, A. Goncalves, and M. Cavazza, "Multi-Agent Path Finding for UAV Traffic Management," pp. 131-139, 2019.

[10] F. O. R. M. Anipulators and M. Robots, .“Oq 0 1985,” pp. 500-505, 1985.

[11] M. Elbanhawi and M. Simic, "Sampling-based robot motion planning: A review," IEEE Access, vol. 2, pp. 56-77, 2014.

[12] R. Jeanne Ross, Weill Peter and David, "STOMP stochastic trajectory optimization for motion planning," J. Chem. Inf. Model., vol. 53, no. 9, pp. 1689-1699, 2006. 
[13] I. Koohi and V. Z. Groza, "Optimizing Particle Swarm Optimization algorithm," Can. Conf. Electr. Comput. Eng., pp. 1-5, 2014.

[14] D. Wang, D. Tan, and L. Liu, "Particle swarm optimization algorithm: an overview," Soft Comput., vol. 22, no. 2, pp. 387-408, 2018.

[15] F. Ho, R. Geraldes, A. Goncalves, M. Cavazza, and H. Prendinger, "Improved Conflict Detection and Resolution for Service UAVs in Shared Airspace," IEEE Trans. Veh. Technol., vol. 68, no. 2, pp. 1231-1242, 2019.

[16] Y. I. Jenie, E. J. Van Kampen, J. Ellerbroek, and J. M. Hoekstra, "Taxonomy of Conflict Detection and Resolution Approaches for Unmanned Aerial Vehicle in an Integrated Airspace," IEEE Trans. Intell. Transp. Syst., vol. 18, no. 3, pp. 558-567, 2017.

[17] D. Šišlák, P. Volf, Š. Kopriva, and M. Pěchouček, "Agentfly: NAS-wide simulation framework integrating algorithms for automated collision avoidance," ICNS 2011 - Integr. Commun. Navig. Surveill. Conf. Renov. Glob. Air Transp. Syst. Proc., pp. 1-11, 2011.

[18] S. Roelofsen, D. Gillet, and A. Martinoli, "Collision avoidance with limited field of view sensing: A velocity obstacle approach," Proc. - IEEE Int. Conf. Robot. Autom., no. 2, pp. 1922-1927, 2017.

[19] P. Kopardekar, K. Bilimoria, and B. Sridhar, "Initial concepts for dynamic airspace configuration," Collect. Tech. Pap. - 7th AIAA Aviat. Technol. Integr. Oper. Conf., vol. 1, no. February 2015, pp. 695-706, 2007.

[20] N. Barnier and C. Allignol, "Combining flight level allocation with ground holding to optimize 4D-deconfliction," Proc. 9th USA/Europe Air Traffic Manag. Res. Dev. Semin. ATM 2011, pp. 636-645, 2011.

[21] S. Chaimatanan, D. Delahaye, M. Mongeau, S. Chaimatanan, D. Delahaye, and M. Mongeau, "Strategic deconfliction of aircraft trajectories To cite this version: Strategic deconfliction of aircraft trajectories," 2013.

[22] V. Duchamp, B. Josefsson, T. Polishchuk, V. Polishchuk, and R. Wiren, "Air Traffic Deconfliction Using Sum Coloring," 2019.

[23] N. Barnier, C. Allignol, N. Barnier, and C. Allignol, "adjustment To cite this version: 4D-Trajectory Deconfliction Through Departure Time Adjustment," 2014.

[24] A. D. Evans, M. Egorov, and S. Munn, "Correction: Fairness in Decentralized Strategic Deconfliction in UTM," pp. 1-17, 2020.

[25] "Blueprint for the sky: The roadmap for the safe integration Balakrishnan, K., Polastre, J., Mooberry, J., Golding, R., and Sachs, P. and 2018. of autonomous aircraft," Airbus UTM, San Francisco, CA, “Airbus_UTM_Blueprint," 2018.

[26] H. P. Dai, D. D. Chen, and Z. S. Zheng, "Effects of random values for particle swarm optimization algorithm," Algorithms, vol. 11, no. 2, pp. 120, 2018.

[27] ICAO, "Unmanned Aircraft Systems Traffic Management (UTM) - A Common Framework with Core Principles for Global Harmonization," UTM Guid., pp. 1-25, 2019. 
$2020-11-18$

Rule-based conflict management for unmanned traffic management scenarios

\author{
Alharbi, Abdulrahman
}

IEEE

Alharbi A, Poujade A, Malandrakis K, Petrunin I, Panagiotakopoulos D \& Tsourdos A (2020)

Rule-based conflict management for unmanned traffic management scenarios. In: 2020

AIAA/IEEE 39th Digital Avionics Systems Conference (DASC), 11-15 October 2020, San

Antonio, USA

https://doi.org/10.1109/DASC50938.2020.9256690

Downloaded from Cranfield Library Services E-Repository 